\title{
5. Über den Einfluß der Röntgenstrahlen auf die Kondensation des Wasserdampfes nach Versuchen von C. Leibfried u. O. Conrad; von Franz Strieder.
}

(Marburger Inauguraldissertationen 1914 und 1915.)

\section{A. Allgemeines über die Kondensation des Wasserdampfes.}

1. Grundzüge der Untersuchungsmethoden.

Die Betrachtung des Einflusses der Röntgenstrahlen auf die Kondensation des Wasserdampfes macht einige Vorbemerkungen über die Kondensationsbedingungen erforderlich. Zunächst sollen die zwei hauptsächlich zum Studium dieser Erscheinungen benutzten Versuchsanordnungen in ihren Grundzügen beschrieben werden. Die zur Kondensation nötige Übersättigung wird bei der Dampfstrahlmethode hervorgebracht durch Ausströmenlassen des Dampfes aus einer Düse. Diese Methode, von R. v. Helmholtz und F. Richarz') zuerst angewandt, ist experimentell sehr einfach, aber von komplizierten Bedingungen in ihrer Wirkung abbängig und infolgedessen $\cdot z u$ Messungen weniger geeignet. Nach der zweiten Methode läßt man ein Dampfvolumen $v_{1}$ sich adiabatisch auf das Volumen $v_{2}$ entspannen und hat dann in $v_{2} / v_{1}$ ein $\mathrm{MaB}$ für die Entspannung. Dieser Vorteil macht die fast ausschließliche Verwendung der „Entspannungsmethode“ für Kondensationsversuche erklärlich. Die Methode wurde angewandt von Coulier und Markart ${ }^{2}$ ), G. Aitken ${ }^{3}$,, J. Kiessling ${ }^{4}$ ), R. v. Helmholtz ${ }^{5}$ ), C. T. R. Wilson ${ }^{6}$ ) u. a.

1) R. v. Helmholtz, Wied. Ann. 32. p. 1. 1887. - R. v. Helm holtz u. F. Richarz, Wied. Ann. 40. p. 161. 1890. - F. Richarz, Wied. Ann. 59. p. 592. 1896.

2) J. Coulier u. Maskart, Journ. de Pharm. et Chim. (4) 22. p. 165. 1875 .

3) J. Aitken, Trans. Roy. Soc. Edinb. 30. p. 337. 1881.

4) J. Kiessling, Abh. d. Naturw. Ver. Hamburg-Altona 8. p. 1. 1884.

5) R. v. Helmholtz, Wied. Ann. 27. p. 527. 1886.

6) C. T. R. Wilson, Philos. Transact. of the Roy. Soc. of London. ser. A. Vol. 189. p. 265-307. 1897. 
2. Die Kondensation reinen Wasserdampfes in Gegenwart von reinen Gasen.

In einem derartigen gesättigten Gemisch über einer Wasseroberfläche würde man bei geringer Temperaturerniedrigung bereits ein Ausfallen des Dampfes in Nebelform erwarten. Da jedoch infolge der geringen Anzahl überzähliger Dampfmolekeln nur sehr kleine Tröpfchen von starker Krümmung entstehen werden, an denen nach den Überlegungen von Sir William Thomson ${ }^{1}$ ) ein höherer Dampfdruck als an ebenen Oberflächen herrscht, so kommt es zu keiner dauernden Kondensation, da die Tröpfehen sofort wieder verdampfen. Nach Untersuchungen C. T. R. Wilsons ${ }^{2}$ ) tritt erst bei achtfacher Übersättigung (etwa 790\%) Nebelbildung ein, da sich hier sehr schnell größere Tropfen von geringerer Krümmung bilden.

3. Die Kondensation des Wasserdampfes in Gegenwart von staubhaltigen Gasen.

$\mathrm{Da}$ die hier vorausgesetzten Staubteilchen Flächen von sehr verschiedener Krümmung, auch ebene, aufweisen, so ist verständlich, daß diese Teilchen schon bei sehr geringen Übersättigungen als Ansatzkerne für kondensierenden Wasserdampf dienen können.

\section{Die Kondensation an Ionen.}

Daß nicht nur Staubteilchen bei Übersättigung die Kondensation des Wasserdampfes einleiten können, hatte bereits 1887 mittels der Dampfstrahlmethode R. v. Helmholtz ${ }^{3}$ ) nachgewiesen. Er setzte den Dampfstrahl den von einer elektrischen Spitzenentladung ausgehenden Ionen aus und fand verstärkte Kondensation. R. v. Helmholtz und F. Richarz ${ }^{4}$ ) wiesen durch zahlreiche Versuche in staubfreier Luft und anderen Gasen mit Dämpfen verschiedener Substanzen einwandfrei nach, daß taisächlich die Ionen als Ansatzkerne wirkten. Die gleichen Erscheinungen erhielten sie bei An-

1) W. Thomson, Phil. Mag. 4. p. 448. 1871. Siehe auch: H. v. Helm holtz, Vorles. Bd. VI. p. 336.

2) C. T. R. Wilson, Phil. Transact. 189. p. 265.1897.

3) R. v. Helmholtz, Wied. Ann. 32. p. 1-19. 1887. 1890.

4) R. v. Helmholtz u. F. Richarz, Wied. Ann. 40. p. 161-202. 
wendung des elektrischen Wechselfeldes als Ionisator. F. Richarzi) entdeckte zuerst die gleiche Wirkung bei Röntgenstrahlen, was C. T. R. Wils on ${ }^{2}$ ) im folgenden Jahre bestätigte. Letzterer fand weiter, daß unterhalb einer Entspannung von $v_{2} / v_{1}=1,25$, entsprechend vierfacher Übersättigung, keine Kondensation eintritt. Dieser Entspannungswert tritt bei einer Druckänderung von $15 \mathrm{~cm}$ Quecksilbersäule ein und wird die Ionengrenze genannt. Von der Ionengrenze ab leiten zunächst bis $v_{2} / v_{1}=1,28$ die negativen Ionen die Kondensation ein; von $v_{2} / v_{1}=1,31$ beginnend bis 1,85 dienen die positiven Ionen als Ansatzkerne. Als weitere Ionisatoren kommen in Betracht: der elektrische Funke, ultraviolette Strahlen und radioaktive Substanzen.

5. Kondensation in Gegenwart chemischer Verbindungen und hygroskopischer Substanzen.

War zur Einleitung der bisher besprochenen Erscheinungen ein Erzwingen der Kondensation durch Übersättigung nötig, so kann man sehen, daß in Gegenwart von Dämpfen des Schwefelsäureanhydrids $\left(\mathrm{SO}_{3}\right)$ u. a., der Wasserdampf ganz freiwillig in Nebelform kondensiert. Die Wirkung hygroskopischer Substanzen wurde zuerst von R. v. Helmholtz und F. Richarz ${ }^{3}$ ) mit der Dampfstrahlmethode nachgewiesen. Daß derartige hygroskopische Substanzen die Kondensation des Wasserdampfes befördern müssen, ist leicht einzusehen. Nitrose Gase als hygroskopische Kerne verursachen einen starken weißen Nebel, nach den. Untersuchungen von E. Barkow ${ }^{4}$ ) und E. Pringal.5)

6. Über das Wesen des „blauen Nebels“.

In Luft oder Sauerstoff kann man beobachten, daß nach eingetretener Kondensation unterhalb der Ionengrenze zu-

1) F. Richarz, Wied. Ann. 59. p. 592-594. 1896. Mitt. d. Naturwiss. Vereins zu Greifswald.

2) loc. cit.

3) R. v. Helmholtz u. F. Richarz, Wied. Ann. 40. p. 161-202; insbesondere 165.1890.

4) E. Barkow, Diss. Marburg 1906, p. 19-23; Ann. d. Phys. IV. 23. p. 32I-324. 1907.

5) E. Pringal, Diss. Marburg 1908, p. 41-54; Ann. d. Phys. IV. 26. p. $740-747.1908$. 
nächst die größeren Nebeltröpfchen infolge Niedersinkens oder Verdampfens verschwinden, dann aber ein feiner blauer Nebel stundenlang sjchtbar bleibt, der nicht verdampft. Es hatten E. Barkow und E. Pringal zwar nachgewiesen, daß durch die Wirkung des Wechselfeldes starke, weiße wolkige Kondensation von nitrosen Gasen erzeugt werde; die Zurückführung des auch von ihnen unter Umständen beobachteten blauen Nebels ouf nitrose Gase begegnete aber, worauf Richarz hinwies, Schwierigkeiten und sollte noch aufgeklärt werden. ${ }^{1}$ ) Die Ionisation durch das Wechselfeld gibt aber nicht so reine Bedingungen wie die durch ultraviolettes Licht oder Röntgenstrahlen. Die auf Anregung von F. Richarz unternommenen Versuche von W. Bieber ${ }^{2}$ ) mi ultraviolettem Licht als Ionisator lieferten einwandfrei den Nachweis, daß die Kerne des blauen Nebels aus Wasserstoffsuperoxyd beständen. Dies hatte auch bereits C. T. R. Wilson ${ }^{3}$ ) angenommen, aber nicht nachgewiesen. C. Leibfried und O. Conrad untersuchten dann näher die durch Röntgenstrahlen hervorgerufene Kondensation.

B. Die Finwirkung der Röntgenstrahlen auf die erzwungene Kondensation des Wasserdampfes unterhalb der Ionengrenze.

1. Spezielle Angaben über den Versuchsplan.

Vor den Untersuchungen C. Leibfrieds war angenommen worden, daß Röntgenstrahlen nur Ionen als Kerne liefern könnten. Die Versuche Leibfrieds ergaben auch unterhalb der Ionengrenze Bildung von Kernen, die sich analog den von $\mathrm{Bieber}$ beobachteten verhielten und schon Kondensation bei der sehr geringen Entspannung um $0,4 \mathrm{~cm}$ veranlaBten.

1) F. Richarz, Marb. Sitzunsberg. Nr. 4 vom 13. Mai 1908.

2) W. Bieber, Diss. Marb. 1911, p. 33-37; Ann. d. Phys. IV. 39. p. 1313-1337. 1912. Bezüglich der Priorität der Untersuchungen Bie bers siehe Meteorolog. Zeitschr. Heft 7. 1914. p. 357. Fußnote 9. Insbesondere auch die Zusammenstellung und Erwähnung der Bieberschen Resultate bereits 1911 vor ihrer Veröffentlichung in A. Wegeners Thermodynamik d. Atmosphäre p. 242 . Anmerkung 4. Leipzig 1911.

3) C. T. R. Wilson, Phil. Transact. London A. Vol. 192. p. 403-453. 1899. 
Es war demnach weiter zu forschen, ob die Röntgenstrahlen nicht auch freiwillige Kondensation hervorrufen könnten, was Conrad ausführte. Da die Versuche Leibfrieds, die Dampfstrahlmethode quantitativ brauchbar zu machen, fehlschlugen, so verwandte er die Entspannungsmethode in einer schon von Bieber benutzten Form.

2. Apparatur zu Versuchen unterhalb der Ionengrenze. (Vgl. Fig. 1.)

Über die Versuchsanordnung sei das folgende mitgeteilt. Als Kondensationsgefä $\beta$ diente ein Rundkolben $A$ von etwa 21 Inhalt, aus Jenaer Glas, dessen Hals $B$ auf einen großen Schliffstopfen $C$ paßte. Letzterer war mit einem zentralen Anschlußrohr mit Hahn $D$ versehen, zwecks Einlassens von Wasser. Außerdem waren in den Schliffstopfen noch drei Glasröhren eingeschmolzen, die sämtlich mit Hähnen nahe an dem Schliffstopfen versehen waren und bis etwa in die Mitte des Rundkolbens reichten. Durch $F$ wurde das Versuchsgas eingeleitet, $E$ führte zur Vakuumeinrichtung, mittels weleher die Entspannungen vorgenommen wurden, $G$ stellte die Verbindung mit dem Manometer $H$ her, welches die Größe der Entspannung angab. Der Schliff am Rundkolben war mit einer konzentrischen Rinne $M$ zur Aufnahme von Quecksilber versehen, um eine sichere Dichtung zu haben. Am Manometer war bei $I$, dem höchsten Punkt, bis zu welchem das Quecksilber bei den vorkommenden Entspannungen steigen konnte, ein Seitenstutzen mit Hahn $K$ angebracht. Hatte man im Versuchsgefä $B$ das Gas kernfrei gemacht, so konnte man kernfreies Gas bis $I$ treiben, war also sicher, daB bei späteren Versuchen aus dem Manometer keine Kerne in das Versuchsgefäß zurückgelangten. Um in letzterem eine Entspannung auszuführen, wurde es mit dem Vorvakuum, einem gläsernen Kugelballon von etwa $40 \mathrm{~cm}$ Durchmesser verbunden, an den ein Manometer angeschlossen war. Mit diesem Vorvakuum war noch ein sogenanntes Hauptvakuum verbunden, bestehend aus fünf Kugelballonen von je $30 \mathrm{~cm}$ Durchmesser. Sollte nun eine Entspannung von bestimmter Größe im Nebelgefäß ausgeführt werden, so wurde der Hahn am Glasrohr $E$ geschlossen, das Vorvakuum mit dem Hauptvakuum in Verbindung gesetzt, bis darin ein durch Vorversuche ermittelter, den ge- 
wünschten Entspannungsdruck etwas übersteigender Unterdruck eingetreten war, dann die Verbindung zwischen Vor-
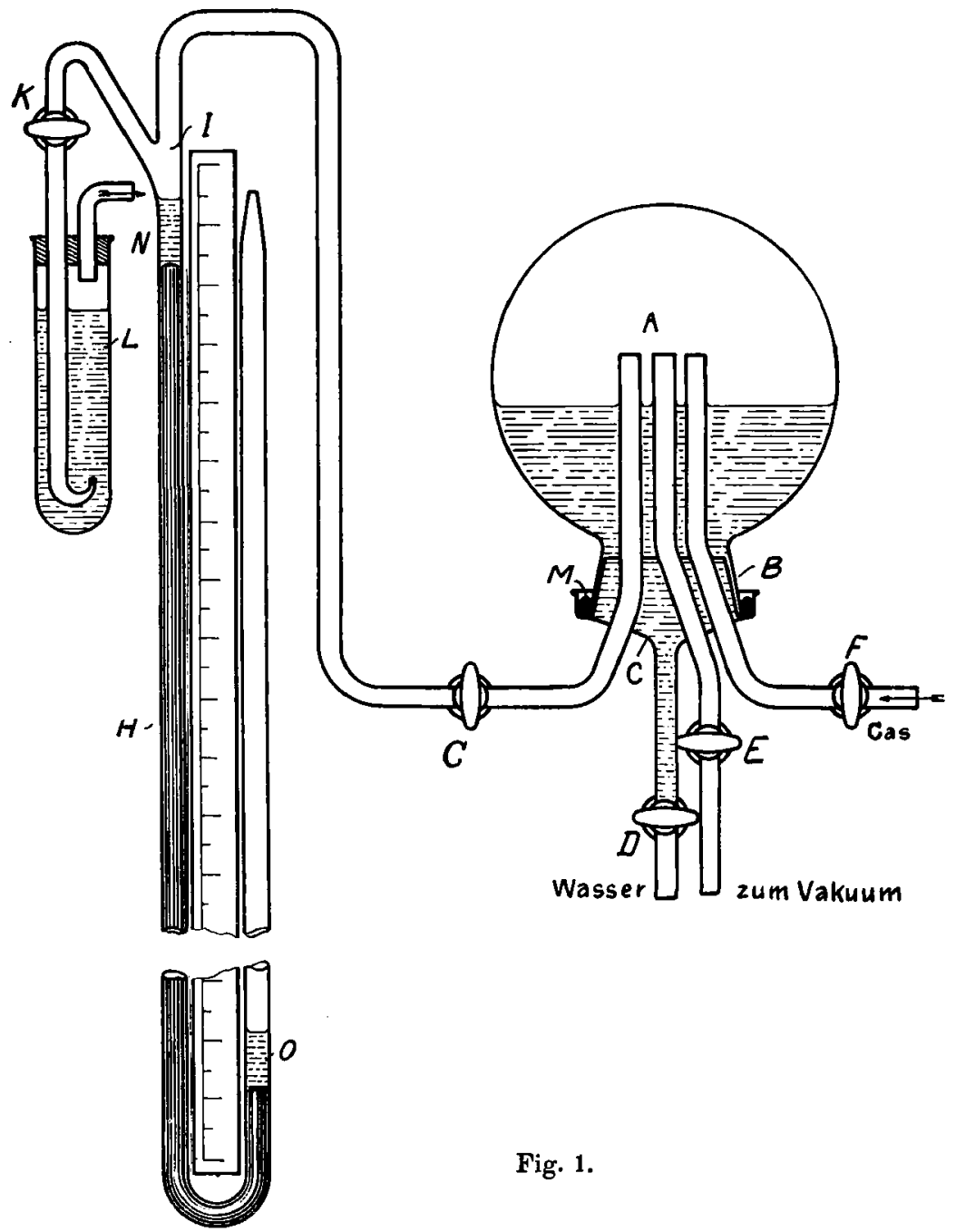

Fig. 1.

und Hauptvakuum unterbrochen und alsdann durch Öffnen des Hahns in $E$ die Entspannung ausgeführt. Der Hahn mußte zu diesem Zweck eine weite Bohrung besitzen. Mittels des 
Manometers $H$ konnte dann die Größe der Entspannung bestimmt werden. Das Nebelgefäß war zu ein Viertel mit luftfreiem destillierten Wasser gefüllt. Eine lichtdicht eingeschlossene Bogenlampe warf mittels eines Kondensators einen Lichtkegel in das Nebelgefä $B$, wodurch die geringste Tropfenbildung gut sichtbar wurde.

\section{Ionisationsapparatur.}

Benutzt wurde eine Intensivstromröhre mit Regeneriervorrichtung, die zur Abblendung der überflüssigen Strahlen in einem mit Bleiblech ausgeschlagenen Holzkasten untergebracht war. Der Bleibelag war zur Vermeidung störender Induktionswirkungen geerdet. Betrieben wurde die Röntgenröhre mittels eines Funkeninduktors. von etwa $35 \mathrm{~cm}$ Schlagweite bei etwa 11-12 Amp. Primärstromstärke. Die Röhre war zum Nebelgefäß so ausgerichtet, daß dasselbe vom Maximum der Strahlung getroffen wurde.

Zunächst sollte die ganze Apparatur zu Kondensationsuntersuchungen in Luft benutzt werden. Die Luft mußte vor Allem möglichst von Kernen gereinigt werden. Sie wurde zu diesem Zweck durch ein Chlorkalziumrohr, alsdann durch ein langes Glaswollefilter durchgesaugt und schließlich in einem Pettenkoferschen Rohr mit Wasserfüllung gesättigt. Die noch im Nebelgefäß vorhandenen Kerne wurden durch eine Reihe von Kondensationen niedergeschlagen.

4. Bei Röntgenbestrahlung von Luft treten Kerne für Kondensation unter der Ionengrenze auf,

Die Versuche ergaben ein Auftreten von Kernen für Kondensation teilweise weit unterhalb der Ionengrenze. Da diese Kondensationen unter ziemlich verschiedenen Umständen eintraten, war ein Einfluß der wechselnden Härte bzw. der verschieden langen Bestrahlungsdauer zu vermuten. In diesen beiden Richtungen wurden die Versuche nunmehr fortgesetzt. Um die Härte der Strahlen zu verändern, wurden Filter aus Zinkblech verschiedener Dicke in den Strahlengang eingeschaltet und dabei gleichzeitig die Bestrahlungsdauer erhöht. Bei jedem Filter wurde zu jeder Bestrahlungsdauer die 
Entspannung bestimmt, bei der noch Kondensation auftrat. Die Ergebnisse sind in den folgenden Tabellen zusammengetragen.

Tabelle 1.

Zinkfilter von $0,46 \mathrm{~mm}$ Dioke.

\begin{tabular}{c|c}
\hline $\begin{array}{c}\text { Dauer der } \\
\text { Bestrahlung }\end{array}$ & $\begin{array}{c}\text { Entspannung in cm } \\
\text { Quecksilber für die } \\
\text { untere Kondensations- } \\
\text { grenze }\end{array}$ \\
\hline \hline 2 Min. & $2,0 \mathrm{~cm}$ \\
3 ," & 1,1, \\
4, & 0,7,
\end{tabular}

Tabelle 2.

Zinkfilter von $0,71 \mathrm{~mm}$ Dicke.

\begin{tabular}{c|c}
\hline \hline $\begin{array}{c}\text { Dauer der } \\
\text { Bestrahlung }\end{array}$ & $\begin{array}{c}\text { Entspannung in cm } \\
\text { Quecksilber für die } \\
\text { untere Kondensations- } \\
\text { grenze }\end{array}$ \\
\hline \hline 2 Min. & $\mathbf{8 , 0} \mathrm{cm}$ \\
$3, "$ & $\mathbf{4 , 0}$, \\
4, & $\mathbf{3 , 0}$,
\end{tabular}

Die Tab. 1 und 2 lassen erkennen, daß bei beiden Filtern mit zunehmender Bestrahlungsdauer die Kondensationsgrenze erniedrigt wird, und zwar bei dem dünneren Filter stärker als bei dem dickeren. Tab. 3 zeigt, daß mit zunehmender Dauer der Bestrahlung schließlich ein Grenzwert für den Eintritt der Kondensation erreicht wird.

Tabelle 3.

Zinkfilter von $0,46 \mathrm{~mm}$ Dicke.

\begin{tabular}{|c|c|}
\hline $\begin{array}{l}\text { Dauer der } \\
\text { Bestrahlung }\end{array}$ & $\begin{array}{l}\text { Entspannung in cm } \\
\text { Quecksilber für die } \\
\text { untere Kondensations- } \\
\text { grenze }\end{array}$ \\
\hline 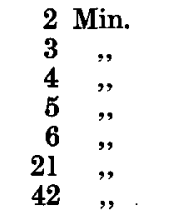 & $\begin{array}{l}2,0 \mathrm{~cm} \\
1,1 \quad, \\
0,7 \quad ", \\
0,4 \quad " \\
0,4 \quad " \\
0,4 \quad, \\
0,4 \quad "\end{array}$ \\
\hline
\end{tabular}


Bei diesen Versuchen lassen sich die beiden Wirkungen der Filter, einerseits durch Absorption der weicheren Strahlen vorzugsweise die härteren zum Kondensationsgefä $B$ gelangen zu lassen, andererseits die Gesamtintensität der Strahlung herabzusetzen, nicht genügend voneinander trennen. Dies durchzuführen würde Gegenstand besonderer Versuche sein. Die erzeugten Kondensationskerne hielten sich sehr lange; nach starken Bestrahlungen konnten sie noch viele Stunden später wahrgenommen werden.

5. In völlig sauerstoffreiem Wasserstoff tritt keine Kondensation unterhalb der Ionengrenze ein.

Der Wasserstoff wurde durch Elektrolyse von 30\% iger aus reinstem Stangennatron hergestellter Natronlauge gewonnen. Als Elektroden dienten Nickelbleche. Zwecks Reinigung des Wasserstoffs von Natronlaugebläschen, die als Kondensationskerne wirken würden, durchströmte das Gas zunächst eine leere Waschflasche, in welcher die Laugebläschen infolge Richtungs- und Geschwindigkeitsänderungen leicht ausfallen. Trotzdem noch mit gerissene Laugeteilchen wurden in einer zweiten Waschflasche mit konzentrierter Schwefelsäure entfernt. Eine dann folgende Röhre mit Natronkalk fing die vom Gasstrom mitgeführte Schwefelsäure ab. Von Sauerstoff wurde das Gas mittels Durchleiten durch ein rotglühendes mit Kupferwolle beschicktes Kupferrohr befreit. Das so gereinigte Gas wurde in einem Gasometer aufgefangen und konnte nach Sättigung mit Wasserdampf in einem Pettenkoferschen Rohre dem Nebelgefäß zugeleitet werden. Nachdem durch eine Reihe von Entspannungen sämtliche im Wasserstoff noch vorhanden gewesenen Kerne beseitigt waren, begannen die Bestrahlungsversuche. Bei Bestrahlungsdauern von etwa 6 Minuten und Entspannungen zwischen 12 und $14 \mathrm{~cm}$ Quecksilber trat niemals Kondensation ein.

6. Durch Röntgenbestrahlung von Luft können Kerne des blauen Nebels entstehen.

Das Eintreten der Kondensation unterhalb der Ionengrenze widerspricht zunächst anscheinend den Feststellungen 
C. T. R. Wil son $\mathbf{s}^{\mathbf{1}}$ ), stimmt aber mit einem Versuchsergebnis von E. Barkow ${ }^{2}$ ) für Luft und einem anderen von W. Bieber ${ }^{3}$ ) bei reinem Sauerstoff überein. Da nun bei diesen Versuchen die Kerne sich stundenlang hielten, nach Wilson aber den Ionen nur eine Lebensdauer von einigen Sekunden zukommt, während Barus ${ }^{4}$ ) ihnen wenigstens noch nach 20 Sekunden einen Einfluß auf den Dampfstrahl zugesteht, so ist man zu der Annahme berechtigt, daß die bei den erwähnten Versuchen auftretenden Kerne nicht als Ionen anzusprechen sind. Vielmehr muß es sich um Teilchen chemischer Verbindungen handeln, die entweder direkt unter dem Einfluß der Röntgenstrahlen zustande kommen oder auch sekundär den primär gebilaeten Ionen ihre Entstehung verdanken. Sind nun einmal unter dem Einfluß der Röntgenstrahlung hygroskopische Kerne entstanden, so werden sie so lange Wasserdampfmolekeln anziehen, bis Dampfdruckgleichgewicht vorhanden ist. Wird nun keine weitere Übersättigung herbeigeführt, so ist das Bestehen solcher Tröpfchen während einer längeren Zeitdauer durchaus verständlich. $\mathrm{Da}$ diese Nebeltröpfchen ähnliche Eigenschaften zeigen wie die von W. Bieber ${ }^{5}$ ) unter dem Einfluß ultravioletten Lichtes nachgewiesenen Wasserstoffsuperoxydkerne, so konnte man schließen, daß auch diese durch Röntgenstrahlen erzeugten Kerne aus $\mathrm{H}_{2} \mathrm{O}_{2}$ bestehen, also denselben, die bei den Versuchen von Bieber den blauen Nebel verursachten und sich ebenfalls durch große Beständigkeit auszeichneten. Auch der Umstand, daß bei den Versuchen in reinem Wasserstoff von C. T. R. Wils on ${ }^{6}$ ), Vincent ${ }^{7}$ ), Bieber ${ }^{8}$ ) und C. Leibfried ${ }^{9}$ ) keine Kernbildung unterhalb der Ionengrenze zu beobachten war, spricht für die obige An-

1) C. T. R. Wilson, Philos. Transact. of the Roy. Soc. of London. Ser. A. Vol. 192. p. 403-453. 1899 und Ser. A. 189. p. 265-307. 1897.

2) E. Barkow, Diss. Marburg 1906. p. 23-25; Ann. d. Phys. IV. 23. p. $317-344.1907$.

3) W. Bieber, Diss. Marburg 1911. p. 37; Ann. d. Phys. IV. 26. p. 727-730. 1908 .

4) C. Barus, Smithsonian Contributions 1309. 1901.

5) W. Bieber, Diss. Marburg 1911. p. 32-35; Ann. d. Phys. IV. 39. p. 1313-1337. 1912.

6) C. T. R. Wilson, loc. cit.

7) R. Vincent, Proc. of the Cambridge Phil. Soc. Vol.12. Part. IV. 1904.

8) W. Bieber, Diss. Marburg 1911.

9) C. Leibfried, Diss. Marburg 1914, p. 23. 
nahme, zumal ja nach W. Bieber ${ }^{\mathbf{1}}$ ) die Kernbildung in reinem Sauerstoff ähnlich verläuft wie in Luft. Der scheinbare Widerspruch zwischen den Versuchen von C. T. R. Wilson und Leibfried bezüglich der Ionengrenze ist wohl folgendermaßen zu erklären: Wilson bestrahlte vermutlich nur so kurze Zeit, aber recht intensiv, daß sich bei ihm nur die primär gebildeten Ionen geltend machten. Leibfried bestrablte länger und erhielt dadurch sekundär chemische Produkte mit Kondensation unter der Ionengrenze.

\section{Freiwillige Kondensation erzeugt durch Röntgenstrahlen in Gegenwart von Bauerstoff.}

\section{Plan der weiteren Untersuchungen.}

Die im Vorigen beschriebenen Versuche von C. Leibfried hatten zwar keine freiwillige Kondensation des Wasserdampfs in Luft ergeben, aber immerhin Kondensationen, deren Kerne mit den durch ultraviolettes Licht erzeugten Kernen des blauen Nebels eine solche Ähnlichkeit zeigten, daß man vermuten konnte, diese Kerne seien bei beiden Versuchen identisch. Da Leibfried zudem eine starke Abhängigkeit der Kondensationsgrenze von der Härte und Dauer der Röntgenstrahlen feststellen konnte und noch dazu schon bei der sehr niedrigen Entspannung von $0,4 \mathrm{~cm}$ Kondensation erhalten hatte, schien es nicht ausgeschlossen, durch Steigerung der Röntgenenergie und Berücksichtigung anderer die freiwillige Kondensation begünstigender Umstände diese auch durch Röntgenstrahlen zu erreichen. Zudem sprach der negative Ausfall der Wasserstoffversuche Leibfrieds auch zugunsten dieser Vermutung. Das Auftreten des blauen Nebels war früher auf sehr verschiedene Ursachen zurückgeführt worden, welche hier wenigstens kurz erwähnt werden müssen. Da nach E. Barkows ${ }^{2}$ ) Versuchen der blaue Nebel in Luft bei Gegenwart von Ozon besonders intensiv auftritt, glaubte er dessen Ursache in den bei der Oxydation des Stickstoffs entstandenen nitrosen Verbindungen gefunden zu haben, die jedenfalls nach ihm die Ursache starker weißer Nebel sind. Übereinstimmend mit ihm machte E. Pringal ${ }^{3}$ )

1) W. Bieber, Diss. Marburg 1911.

2) E. Barkow, Ann. d. Phys. 23. p. 332. 1907.

3) E. Pringal, Diss. Marburg 1908. p. 50. 
auch Stickoxyde für das Auftreten des blauen Nebels verantwortlich. Ein Bericht über diese Untersuchungen hat K. Stu chtey in der Marburger Gesellschaft zur Beförderung der gesamten Naturwissenschaften erstattet. ${ }^{1}$ )

Aus Versuchen von Meißner ${ }^{2}$ ), R. v. Helmholtz und F. Richarz ${ }^{3}$, E. Barkow ${ }^{4}$, E. Pringal ${ }^{5}$ ), Leithäuser, R. $P o h 1^{6}$ ) und W. Bieber ${ }^{7}$ ) ging hervor, daß Ozon selbst nicht die Ursache des blauen Nebels sei, weder in statu nascendi, noch in gealtertem Zustande. Da nun Prof. F. Richarz ${ }^{8}$ ) die Oxydation des Stickstoffs bei der Bildung des blauen Nebels für nicht wahrscheinlich hielt, auch Pringal gefunden hatte daß der blaue Nebel um so stärker sei, je stickstoffreier und je sauerstoffreicher ein Gas sei, so untersuchte W. Bieber den blauen Nebel genauer und konnte $\mathrm{H}_{2} \mathrm{O}_{2}$ als Kernsubstanz feststellen, sowohl bei Einwirkung von Ozon als auch von ultraviolettem Licht. Im ersten Fall konnte man sich die $\mathrm{H}_{2} \mathrm{O}_{2}$ Bildung nach folgenden Reaktionen denken:

$$
\begin{aligned}
& \mathrm{O}_{3}=\mathrm{O}_{2}+\mathrm{O} \\
& \mathrm{O}+\mathrm{H}_{2} \mathrm{O}=\mathrm{H}_{2} \mathrm{O}_{2} .
\end{aligned}
$$

Im zweiten Fall ließ sich die Entstehung von $\mathrm{H}_{2} \mathrm{O}_{2}$ durch Dissoziation des Sauerstoffs folgendermaßen erklären:

$$
\begin{gathered}
\mathrm{O}_{2}=\mathrm{O}+\mathrm{O}, \\
\mathrm{H}_{2} \mathrm{O}+\mathrm{O}=\mathrm{H}_{2} \mathrm{O}_{2}, \quad \mathrm{O}_{2}+\mathrm{O}=\mathrm{O}_{3},
\end{gathered}
$$

das außerdem entstandene Ozon konnte dann nach der vorher erörterten Reaktion $\mathrm{H}_{2} \mathrm{O}_{2}$ liefern. Die Versuche von $\mathrm{Bieber}$ und Leibfried, auch mittels Röntgenstrahlen diese Reaktionen

1) K. Stuchtey, Ber. d. Ges. zur Bef. d. ges. Naturwissensch. Marburg 1910. Nr. 5. 1863.

2) Mei ßner, Untersuchungen über den Sauerstoff. p. 309. Hannover

3) R. v. Helmholtz u. F. Richarz, Wied. Ann. 40. p. 167, 175, 194/195. 1890 .

4) E. Barkow, Ann. d. Phys. 23. p. 336. 1907.

5) E. Pringal, Diss. Marburg 1908. p. 58. 1908.

6) C. Leithä user u. R. Pohl, Ver. d. Deutsch. Phys. Ges. 10. p. 253.

7) W. Bieber, Diss. Marburg 1911. p. 17-20. Vgl. diese vorlieg. Abh. oben p. 990.

8) F. Richarz, Sitz.-Ber. d. Ges. z. Bef. d. ges. Naturw. Marb. Nr. 4 v. 13. 5. 1908 . 
einzuleiten, d.h. freiwillige Kondensation zu erhalten, verliefen ergebnislos, was an Verwendung zu schwacher Röntgenstrahlen liegen konnte. O. Conrad unternahm es auf Anregung von Prof. F. Richarz für Luft, Sauerstoff und Stickstoff diese Versuche möglichst zu einer Entscheidung zu bringen.

2. Anordnung zur Untersuchung freiwilliger Kondensation.

In den Versuchen sollte die Methode der adiabatischen Entspannung benutzt werden. Auf Anraten von K. Stuchtey wurde eine den bisher beschriebenen Anordnungen gegenüber wesentlich verbesserte Ausführungsform gewählt, die in ihren Grundzügen von C. T. R. Wils on ${ }^{\mathbf{1}}$ ) ausgebildet wurde.

Die Versuchseinrichtung bestand aus dem kugelförmigen NebelgefäB $A$ (vgl. Fig. 2) von $16 \mathrm{~cm}$ Durchmesser, welches unten durch den Schliff mit Quecksilberdichtung $Y$ verschlossen war. Diesen Schliff durchsetzte ein Glasrohr mit dem Hahn $E$, zur Ableitung der Versuchsgase bestimmt. Das Rohr $F$ diente zum Einführen bzw. Ablassen des Wassers in $A . A$ stand durch das $2 \mathrm{~cm}$ weite Glasrohr $D$ mit dem zylindrischen Gefäß $B$ in Verbindung. Den Boden desselben bildete ein Gummistopfen $W$, durch welchen das oben mit einer schalenförmigen Verbreiterung versehene Rohr $G$ eintrat. Über $G$ war ein „Kolben“ $C$, ein oben geschlossener Glaszylinder gestülpt, der sich zwischen der Wand von $B$ und der Verbreiterung von $G$ frei bewegen konnte. $B$ war etwa zur Hälfte mit Quecksilber gefüllt, auf dem sich eine Schicht Wasser befand. $A$ und $B$ standen also stets miteinander in Verbindung, waren aber vom Inneren von $C$ durch das Quecksilber getrennt. Das Rohr $G$ stand durch den Hahn $L$ mit der AuBenluft in Verbindung, außerdem durch das Rohr $V$ mit dem Vorvakuum $S$ und weiter mit dem Hauptvakuum $U$. Zwischen $G$ und $V$ war das Entspannungsventil $H$ von großem Querschnitt eingebaut, im wesentlichen aus einem federbelasteten Kolben $K$ mit Knopf $I$ bestehend, $\operatorname{der} G$ von $V$ abschließen konnte. Zwischen $S$ und $U$ befand sich ein Manometer. Die Wirkungsweise der Apparatur läßt sich nun bereits übersehen. Herrseht in $A$ und $B$, sowie im Inneren

1) C. T. R. Wilson, Phil. Transact. London A. Vol. 192. p. 405.1899. 


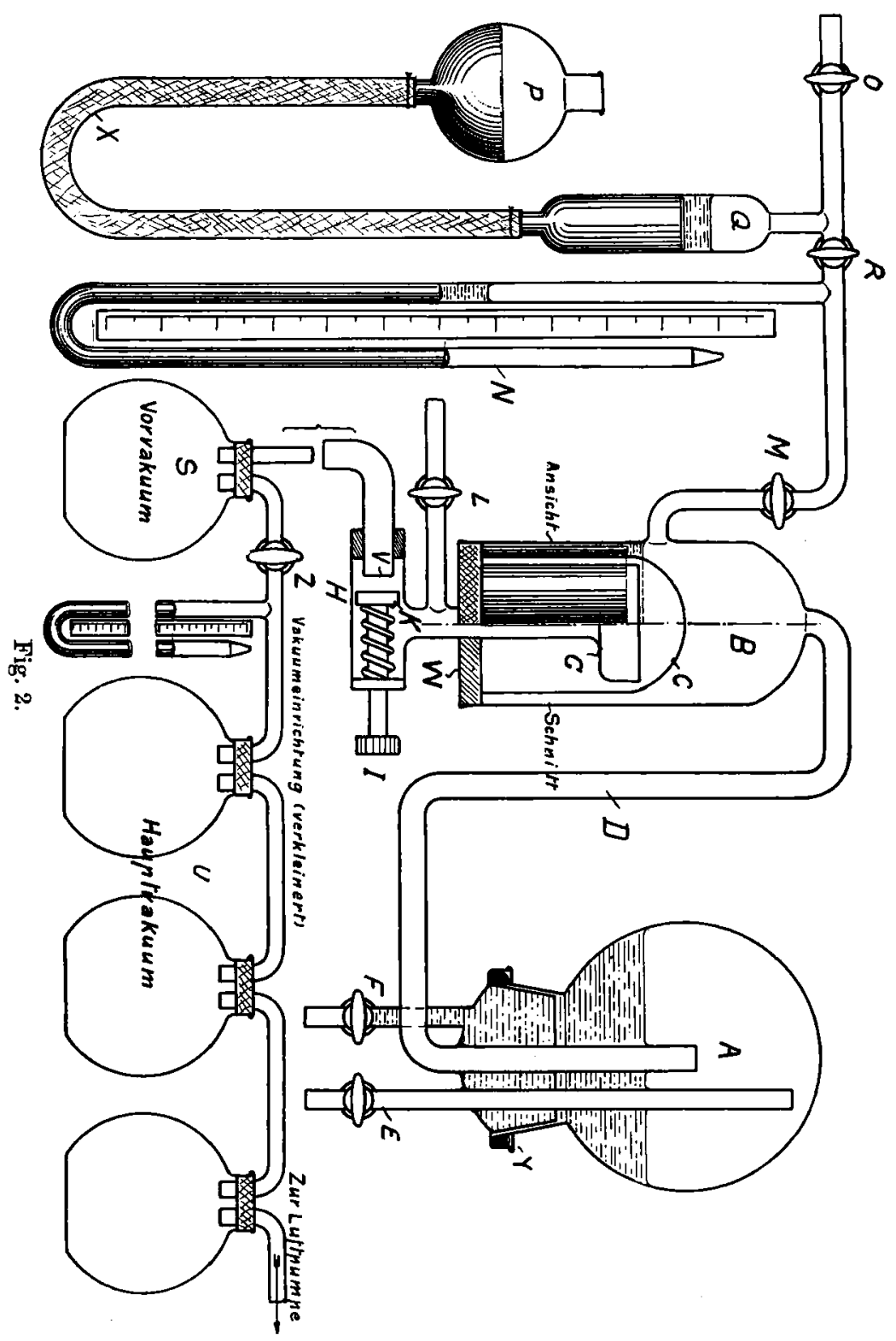

von $C$ Atmosphärendruck, so nimmt $C$ infolge des Auftriebs im Quecksilber einen bestimmten Stand ein. Verbindet man das 
Innere von $C$ mittels des Ventils $H$ mit dem Vakuum, so wird der Kolben $C$ schnell bis auf $W$ herabgedrückt, in $A$ und $B$ also eine Entspannung hervorgerufen, die von dem Stand von $C$ vor der Entspannung abhängt. Um letztere verändern zu können, muß durch Regelung des Druckes in $A$ und $B$ der Stand von $C$ vor der Entspannung festgelegt werden. Je höher $C$ steht, um so größer wird die Entspannung und umgekehrt. Die erwähnte Druckregelung erfolgt durch ein Gefä $\beta$, welches an der zu $B$ führenden Leitung mit Hahn $M$ angebracht ist; $Q$ steht durch einen Schlauch $x$ mit dem Hubgefä $B P$ in Verbindung; $Q$ und $P$ enthalten eine bestimmte Quecksilbermenge. Durch Heben oder Senken von $P$ kann man in $B$ und $A$ den gewünschten Druck erzeugen. Zieht man den Kolben $C$ mittels des Vakuums auf seinen tiefsten Stand, stellt in $A$ und $B$ einen am Manometer $N$ bestimmbaren gewünschten Unterdruck her, schließt $A$ und $B$ durch Hahn $R$ von $Q$ ab, so wird nach Öffnen von $L$ der Kolben $C$ steigen, bis in $A$ und $B$ Atmosphärendruck herrscht. SchlieBt man nun $L$ und verbindet $C$ mit dem Vakuum, so tritt wieder in $A$ und $B$ der vorher an $N$ abgelesene Unterdruck ein, also Entspannung gegenüber dem Atmosphärendruck.

Die Leitung mit Hahn $O$ dient noch zum Einführen des Gases in $A$ und $B$. Der Hauptvorteil dieser Apparatur liegt im Gegensatz zu der früher beschriebenen darin, daß man mit einer einmal eingefüllten Gasmenge beliebig viele Versuche machen kann, da Nebelgefäß und Vakuum durch den Kolben $C$ getrennt sind. Da keine Hähne am Nebelgefäß betätigt werden, können keine Verunreinigungen durch Fettdämpfe eintreten, außerdem ist der geringe Bedarf an Gas bei schwer rein erhältlichen Gasen wichtig.

3. Einfluß der Art der Reinigung des Versuchsgases.

Zunächst wurde Luft mittels Chlorkalzium und Wattefilter möglichst kernfrei gemacht, dann durch Entspannungen über der Ionengrenze alle noch vorhandenen Kerne beseitigt, so daß keine Kondensation mehr eintrat. Wurde diese Luft nun Röntgenstrahlen ausgesetzt, so trat unterhalb der Ionengrenze wieder Kondensation ein. Wurde andererseits ungereinigte Zimmerluft im Nebelgefäß nur darch vielfach wiederholte Entspannungen gereinigt, so zeigte sich bei Röntgenbestrahlung dasselbe Resultat. Letztere Art der Reinigung genügt also bereits. 
4. Erniedrigung der Kondensationsgrenze mit der Bestrahlungsdauer bis zur freiwilligen Kondensation.

Zu diesen Versuchen wurde dieselbe Röntgenapparatur wie bei den Versuchen von C. Leibfried benutzt, jedoch die Röntgenröhre dem Nebelgefäß auf $\mathbf{3} \mathrm{mm}$ genähert. Als Filter für die Röntgenstrahlen dienten Kupferbleche. Ohne Kupferfilt $\in \mathbf{r}$ wurde bei einer Primärstromstärke von $12 \mathrm{Amp}$. freiwillige Kondensation erreicht. Die Versuche sind in Tab. 4 zusammengestellt.

\section{Tabelle 4.}

Bestrahlung ohne Cu-Blech.

Primärstromstärke $12 \mathrm{Amp}$.

\begin{tabular}{|c|c|}
\hline Bestrahlungszeit & Expansion \\
\hline 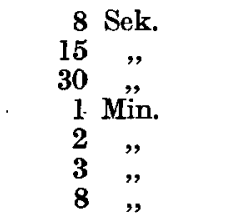 & $\begin{array}{l}1,7 \mathrm{~cm} \\
1,2 \quad, \\
0,8 \quad " \\
0,4 \quad " \\
0,4 \quad " \\
0,4 \text { ", } \\
\text { freiwillig }\end{array}$ \\
\hline
\end{tabular}

Die weiteren Versuche mit Filtern von zunehmender Dicke lassen erkennen, daß die Kondensationsgrenze nach oben rückt. Schon bei dem $0,05 \mathrm{~mm}$ dicken Filter war keine freiwillige Kondensation mehr zu erreichen. Die Resultate finden sich in den Tabb. 5, 6 u. 7.

\section{Tabell e 5 .}

Bestrahlung mit Cu-Blech 0,05 mm.

Primärstromstärke $12 \mathrm{Amp}$.

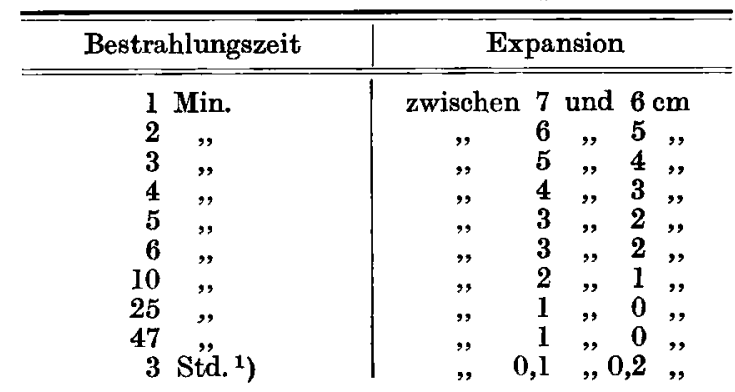

1) Diese lange Bestrahlung von 3 Stunden war natürlich nur möglich bei $\mathrm{Zwischenpausen}$ von 10-15 Minuten. 
Einfluß der Röntgenstrahlen auf die Kondensation usw. 1003

Tabelle 6.

Bestrahlung mit Cu-Blech 0,09 mm. Primärstromstärke 12 Amp.

\begin{tabular}{|c|c|c|c|}
\hline Bestrahlungszeit & $\mathbf{E x}_{1}$ & pansion & \\
\hline $\begin{array}{rc}1 & \text { Min. } \\
2 & \Rightarrow \\
3 & , \\
4 & , \\
5 & \prime \\
6 & \Rightarrow \\
7 & , \\
8 & , \\
15 & \Rightarrow\end{array}$ & $\begin{array}{c}\text { zwischen } \\
\text { ", } \\
\text { " } \\
" \\
" \\
" \\
\text { " }\end{array}$ & 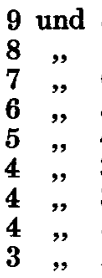 & $\begin{array}{ll}8 & \mathrm{~cm} \\
7 & " \\
6 & \prime \\
5 & " \\
4 & " \\
3 & " \\
3 & " \\
3 & " \\
2 & "\end{array}$ \\
\hline
\end{tabular}

Tabelle 7.

Bestrahlung mit Cu-Blech 0,14 mm. Primärstromstärke 12 Amp.

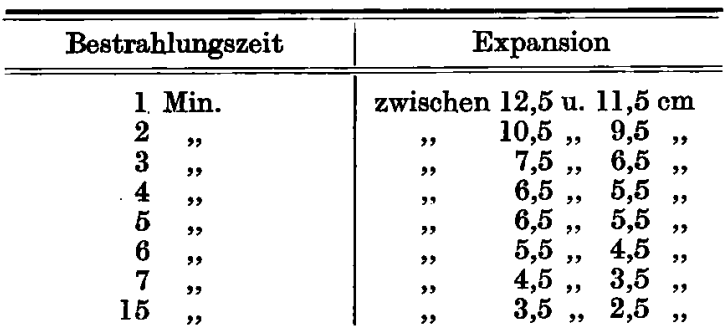

5. Abhängigkeit der freiwilligen Kondensation von der Intensität der Strahlen.

Bei Verminderung der dem Induktor zugeführten Primärenergie wurde die zur freiwilligen Kondensation erforderliche Bestrahlungsdauer größer, wie Tab. 8 zeigt (s. Fig. 6, Kurve a).

$$
\text { Tabelle } 8 .
$$

$\begin{array}{rcc}\text { Bestrahlungszeit } & \text { Kondensation } & \text { Primärstromstärke } \\ 3,5 \text { Min. } & \text { freiwillig } & 13 \\ 8,0, " & , & 12 \\ 11,5, " & , & 11 / 2 \\ 16.0,, & , & 11\end{array}$

Da bei diesen Versuchen die Röntgenröhre ausgewechselt werden mußte, mußten die auf Tab. 4 und 5 zusammengestellten Versuche wiederholt werden (Tabb. 4a u. 5a). 
Tabelle 4 a.

Bestrahlung ohne Cu-Blech.

Primärstromstärke 12 Amp.

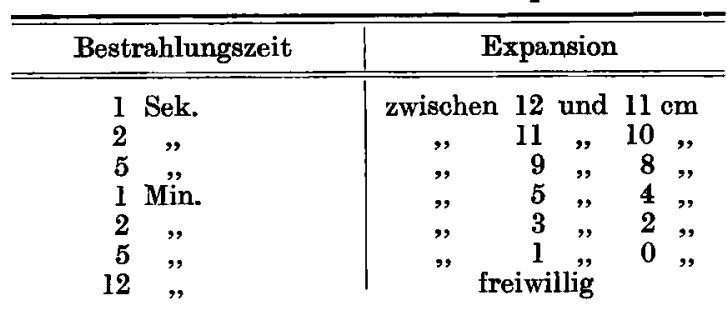

Tabelle 5a.

Bestrahlung mit Cu-Blech $0,05 \mathrm{~mm}$.

Primärstromstärke 12 Amp.

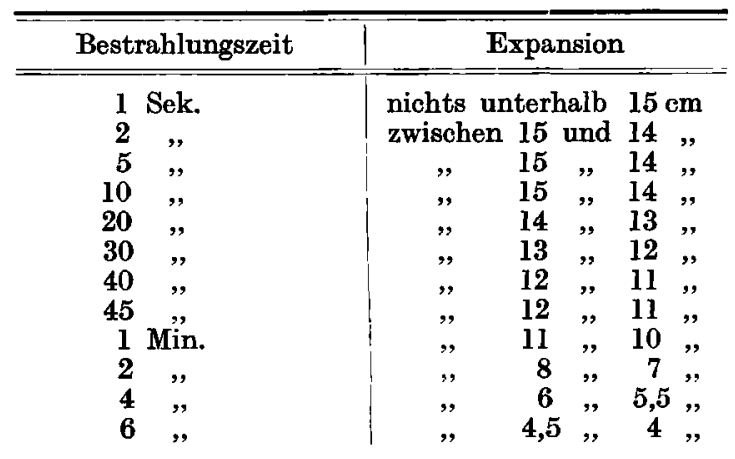

Die Versuche zeigten im wesentlichen denselben Verlauf, nur war die kondensierende Wirkung der neuen Röhre schwächer; hier trat bei der Primärstromstärke von 12 Amp. ohne Filter die freiwillige Kondensation erst nach 12 Min. auf statt nach 8 Min., wie bei der ersten Röhre. (Vgl. Fig. 3, entsprechend Tabb. 4 u. 4a.) Die Versuche der Tabb. 5, 6 u. 7 sind in Fig. 4, die der Tab. 5a in Fig. 5a, die der Tabb. 8 und 9 in Fig. 6 dargestellt. Die Kurven können alle ungezwungen rechts unten so verlängert werden, da $B$ der Bestrahlungsdauer 0 die Entspannung $15 \mathrm{~cm}$ entspricht, d. h. daß die anfängliche Wirkung der Röntgenstrahlen Kondensation bei der Ionengrenze ergibt, welchen Fall C. T. R. Wils on allein beobachtet hat. Dieser Anschluß behebt den scheinbaren Widerspruch, wie oben p. 997 auseinandergesetzt. 
Einfuß der Röntgenstrahlen auf die Kondensation usw. 1005

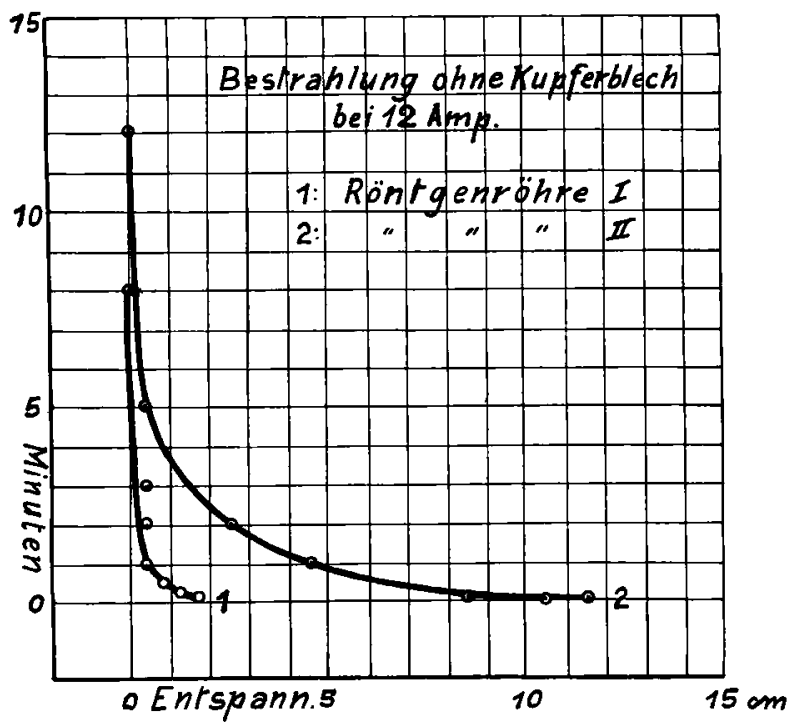

Fig. 3. (Tabb. 4 u. 4 a.)

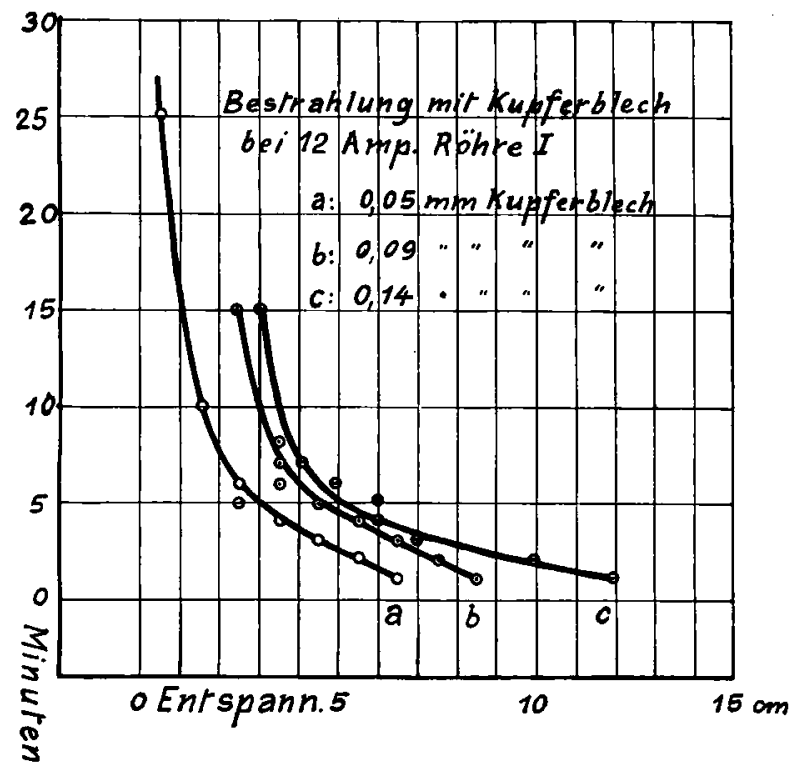

Fig. 4. (Tabb. 5, 6 u. 7.)

Annalen der Physik. IV. Folge. 46. 


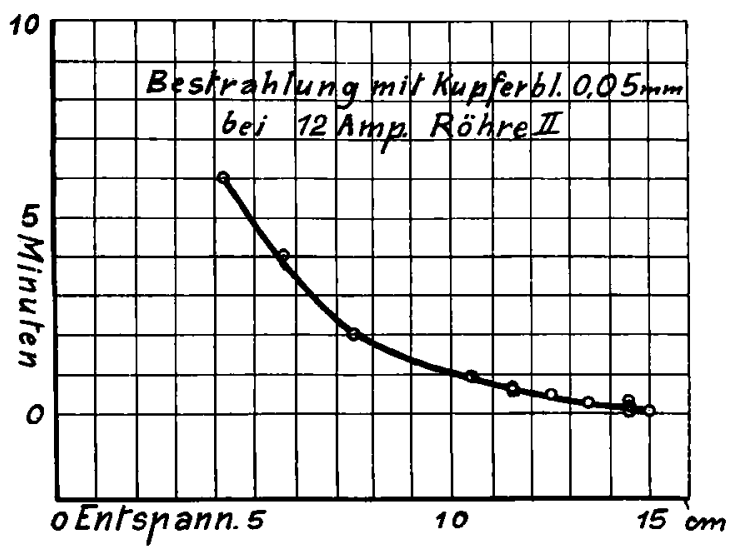

Fig. 5. (Tab. 5a.)

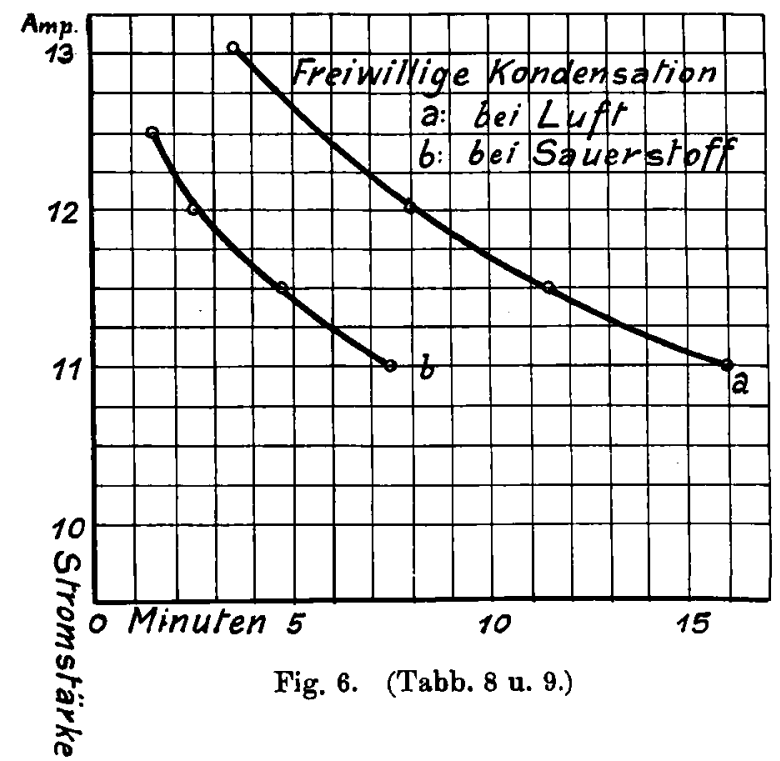

6. In reinem Sauerstoff entstehen Kerne der freiwilligen Kondensation leichter als in Luft.

War so erstmalig freiwillige Kondensation in Luft erhalten worden, so lag es nun nahe, die Versuche mit reinem Sauerstoff zu wiederholen. Der Sauerstoff wurde durch Elektrolyse von 
verdünnter Schwefelsäure zwischen Platinelektroden erhalten, mittels konzentrierter $\mathrm{H}_{2} \mathrm{SO}_{4}$ getrocknet, weiter durch ein Rohr mit Natronkalk und ein solches mit glühendem Kupferoxyd zur Beseitigung von Wasserstoff geleitet und im Pettenkoferschen Rohr angefeuchtet. Der Sauerstoff wurde mit Pyrogallol und Kalihydrat' ) in einer Gaspipette untersucht und als völlig rein erkannt. Bei Röntgenbestrahlung erhielt $O$. Conrad entsprechende Resultate wie für Luft, also Erniedrigung der Kondensationsgrenze mit zunehmender Bestrahlungsdauer bis zur freiwilligen Kondensation. Wie vorauszusehen war, wirkte die Ionisation stärker als bei Luft. (Vgl. die Tabb. 8 u. 9 und die Kurven $a$ und $b$ auf Fig. 6.)

$\begin{array}{ccc} & \text { Tabelle 9, } \\ \text { Bestrahlungszeit } & \text { Kondensation } & \text { Stromstärke } \\ 11 / 2 & \text { freiwillig } & 12^{1 / 2} \\ 21 / 2 & " & 12 \\ 4^{3 / 4} & " & 11^{1 / 2} \\ 71 / 2 & " & 11\end{array}$

Die einzelnen Nebeltröpfchen im Sauerstoff konnten nicht unterschieden werden im Gegensatz zu dem Nebel bei den Versuchen in Luft. Dicse freiwillig auftretenden Nebel, ebenso diejenigen, welche man nach zu früh unterbrochener Bestrahlung dureh Entspannung unterhalb $15 \mathrm{~cm}$ erzwingen konnte, zeigten die mehrfach beschriebene blaue Färbung und die lange Lebensdauer der Tröpfchen. Wurde nach einer Bestrahlung erst nach 2 Tagen erzwungene Kondensation herbeigeführt, so glich der entstandene Nebel völlig dem vorbeschriebenen. Bezüglich des Finflusses der Filter sei auf die Ausführungen auf p. 995 hingewiesen.

7. In reinem Stickstoff ist kein blauer Nebel und keine freiwillige Kondensation zu erhalten.

Diese Versuche machten es in hohem Grade wahrscheinlich, da $B$ die blauen Nebel in Luft und Sauerstoff ihre Entstehung dem von $W$. Bieber nachgewiesenen $\mathrm{H}_{2} \mathrm{O}_{2}$ verdankten. Es war nun erforderlich, festzustellen, ob in einem Gase, wo $\mathrm{H}_{2} \mathrm{O}_{2}$ nicht entstehen konnte, ebenfalls Nebel der beschriebenen Art zu beobachten waren. Als Versuchsgas diente hierzu Stickstoff,

1) Travers. Experiment. Unters. von Gasen. p. 83. Braunsohweig 1905. 
der durch Erhitzen von barytfreiem Ammoniumnitrit hergestellt wurde. Das Gas wurde getrocknet, durch Ferrochloridlösung, 30proz. Kalilauge und Überleiten durch glühende Kupferwolle von Stickoxyden befreit. Dieser sorgfältig gereinigte Stickstoff ergab bei Bestrahlungen bis zu 30 Minuten weder freiwillige, noch erzwungene Kondensation unterhalb der Ionengrenze. Erzwungene Kondensation oberhalb der Ionengrenze trat ein, wie man auch erwarten mußte.

\section{Die Wirkung der Röntgenstrahlen bei der Frzeugung von $\mathrm{H}_{2} \mathrm{O}_{2}$-Kernen ist analog der Wirkung ultravioletten Lichtes.}

Hält man die Versuche von $O$. Conrad zusammen mit dem negativen Ergebnis $d \in r$ Wasserstoffversuche unterhalb der Ionengrenze bei C. Leibfried, so kann man wohl mit Sicherheit behaupten, daß das Entstehen der blauen Nebel an das Vorhandensein von Sauerstoff gebunden ist. Nachdem nun für die durch ultraviolettes Licht hervorgerufenen Kerne des blauen Nebels durch W. Bieber. ${ }^{1}$ ) $\mathrm{H}_{2} \mathrm{O}_{2}$ als Kernsubstanz chemisch nachgewiesen ist, darf man wohl als sicher annehmen, daß die durch Röntgenstrahlen erzeugten Kerne, welche dieselben Erscheinungen hervorrufen, ebenfall ${ }_{3}$ als $\mathrm{H}_{2} \mathrm{O}_{2}$ anzusehen sind. Den Mechanismus dieser $\mathrm{H}_{2} \mathrm{O}_{2}$-Erzeugung kann man sich so erklären, daß die $\mathrm{O}_{2}$-Molekeln durch die Röntgenstrahlen in O-Atome gespalten werden, die dann mit $\mathrm{H}_{2} \mathrm{O}$ zusammen $\mathrm{H}_{2} \mathrm{O}_{2}$ bilden. Einen Beweis für die Wirkungsgleichheit der beiden Strahlungsarten liefert O. Conrad durch folgenden Versuch. Es wurde kernfreie Luft zuerst 1 Minute mit ultraviolettem Licht, dann $1 \frac{1}{2}$ Minuten mit Röntgenstrahlen bestrahlt, bis freiwillige Kondensation eintrat; bestrahlte er zuerst $1 \frac{1}{2}$ Minuten mit Röntgenstrahlen, dann 1 Minute mit ultraviolettem Licht, so erhielt er dasselbe Ergebnis. Es müssen also, da die Reihenfolge ohne Einfluß blieb, die durch beide Strahlungsgattungen erzeugten Kerne dieselbe Wirkung haben. Des weiteren stellte $O$. Conrad fest, daß in reinem Wasserdampf in Abwesenheit von Sauerstoff keine freiwillige Kondensation durch Bestrahlung zu erhalten ist.

Physikal. Institut der Universität Marburg/H., März 1915.

1) W. Bieber, Diss. Marburg 1911, S. 33-37 und l. c.

(Eingegangen 10. März 1915.) 